\title{
Welche Veränderungen kann der Klimawandel für Pollenflug und Pollenbelastung allergener Pflanzen bringen?
}

\section{Das „Wärmejahr" 2018 in Linz (Oberösterreich) im langjährigen Trend als mögliches Zukunftsmodell}

HerWig AE Schinko', Bernd LampreChT², Roland SChmidt ${ }^{3}$

'Allgemeines Krankenhaus der Stadt Linz, Kepler Universitätsklinikum, Med Campus III, Altenberg/Linz, Österreich; ${ }^{2}$ Pneumologie, Kepler Universitätsklinikum, Med Campus III, Linz, Österreich; ${ }^{3}$ Pollenwarndienst Oberösterreich, Mondsee, Österreich

\section{Schlüsselwörter \\ Klimawandel, allergene \\ Pflanzen, Pollen- flug, Belastungs- szenarien, Linz (Oberösterreich)}

\section{Eingang}

12. Dezember 2019

\section{Annahme}

5. August 2020

Englische Fassung https://link.springer. com/journal/40629

\section{Zusammenfassung}

Hintergrund: Global vollzieht sich ein Klimawandel. Pollenallergien nehmen seit Mitte des letzten Jahrhunderts zu. Im Außenraum sorgt die Sensibilisierung gegenüber Pollenallergenen für die höchste Prävalenz von Allergien an Augen und Atemwegen. Daraus ergeben sich folgende Fragen:

1. Wie manifestiert sich der Klimawandel lokalregional und ist das Temperatur- und Niederschlagsverhalten von 2018 exzeptionell?

2. Wie wirken sich geänderte meteorologische Bedingungen auf Pollenflug und Pollenbelastung aus?

Methoden: Es werden die Pollenflugdaten - bezogen von der Pollenmonitoring-Station Linz, Oberösterreich - der wichtigsten allergenen Pflanzen ausgewertet. Dabei wird das Jahr 2018 den Jahren 1993 bis 2017 gegenübergestellt. Mithilfe statistischer Methoden wird der Einfluss meteorologischer Parameter auf den Pollenflug analysiert.

Ergebnisse: Der Klimawandel ist auch regional nachzuweisen. Das regional gemäßigte Klima verschiebt sich in Richtung wärmere und trockenere (semi-aride) Wetterlagen.

Präsaisonale meteorologische Faktoren bestimmen kumulativ Blütenentwicklung, Blüte und Pollenflug (PF). PF-Beginn (PFB) und PF-Dauer von Hasel, Erle, Birke und Gräsern folgen anderen Mechanismen als die saisonale Pollenproduktion (SPIn). Das Modelljahr 2018 bietet durch seinen hybriden Charakter Erklärungen für unterschiedliche Pollenflug- und Belastungsszenarien. Für Hasel (Corylus), Erle (Alnus) und Birke (Betula) ist die
Koinzidenz von kumulierter mittlerer Tageswärme $\left(\mathrm{MTW}_{\mathrm{kumul}}\right)$ und artspezifischer Schwelle der Tageshöchsttemperatur (THT) für den PF-Beginn notwendig. Der früheste war 2018. Frost $\left(\mathrm{MTF}_{\mathrm{kumul}}\right)$ verzögert den PF-Beginn. Präsaisonaler Frost beziehungsweise kühle Temperaturen stimulieren die SPIn von Erle und Birke, während die Pollenproduktion der Hasel wärmeabhängig ist. Wärme verlängert die PF-Dauer der Frühblüher. Hitze - kombiniert mit Dürre - verkürzten diese 2018 bei der Birke. Die relative Feuchte (RF) ist hoch signifikant mit dem Blühbeginn der Gräser korreliert. Wärme und Trockenheit führten 2018 zum frühesten PF-Beginn der Gräser seit 1993. Langfristig nehmen ihre SPIn und Haupt-Pollen-Peaks mit der Trockenheit ab. Schlussfolgerung: Die Annahme, dass die Klimaerwärmung in 26 Jahren zu einer Intensivierung des PF allergener Pflanzen führt, lässt sich für Linz nicht bestätigen. Da die Pollenbelastungen bei Trendanalyse mehrheitlich nicht zu-, sondern abgenommen haben, ist nach anderen Erklärungen für die Zunahme von Pollenallergien und Pollensensibilisierungen zu suchen.

Zitierweise: Schinko HAE, Lamprecht B, Schmidt R. How will climate change alter the dynamics of airborne pollen and pollen load of allergenic plants? The "exceptionally warm year" 2018 in Linz (Upper Austria) - a potential model for the future based on long-term trends. Allergo J Int 2021;30:96-108 https://doi.org/10.1007/s40629-020-00152-4 


\section{Hintergrund}

Pollen zählen zu den wichtigsten Allergen-Carriern im Außenraum. In Österreich hängen Sensibilisierung und Allergie zumindest bei Kindern von der Pollenbelastung ab [1], andererseits wurden in urbanen schadstoffbelasteten Gebieten allergenspezifische IgE bei 37,8\% gegenüber 25,6\% in ländlichen Gebieten gefunden [2]. Bei 501 unselektionierten Jugendlichen im Alter von 12 bis 21 Jahren in Salzburg zeigten rezent $53,5 \%$ eine Sensibilisierung gegenüber Pollenallergenen, 26,5\% gegenüber Phl p 1 (Grasallergen Gruppe 1) und 16,3\% gegenüber Bet v 1 (Hauptallergen der Birke). Die Prävalenz der Pollensensibilisierung betrug 41,7 \% und war von Birke und Gras dominiert [3]. Wegen progressiver Klimaerwärmung, beschleunigt seit 1980, und Anstieg des $\mathrm{CO}_{2}$ in der Luft im vergangenen Jahrhundert von circa 300 auf über 400 ppm (parts per million) wird eine Zunahme der Pollenbelastung erwartet [4]. Vergleiche der Pollenkonzentrationen allergener Pflanzen in der Luft verschiedener Stationen Europas zeigen keinen übereinstimmenden Anstieg [5]. Unterschiedliche Trends bei allergenen Frühblühern und krautigen Sommerblühern (z. B. relativ konstant bei Gräsern, fallend bei Beifuß) tragen zum heterogenen Bild des Einflusses des Klimawandels bei [6]. Die
Analyse des regionalen Klimas und parallel des Pollenflugs allergener Pflanzen - Erle, Hasel, Birke und Gräser - der letzten 25 Jahre in Linz (Oberösterreich) sowie des „Modell-Wärmejahres 2018“ sollten diese Hypothesen überprüfen lassen.

Das Jahr 2018 war nach der Zentralanstalt für Meteorologie und Geodynamik in Wien (ZAMG) das wärmste Jahr in der 251-jährigen Messgeschichte (seit 1768 lagen von den 20 wärmsten Jahren 14 in den 2000er-Jahren). Es ist charakterisiert durch das höchste Temperatur-Jahresmittel von $12,2^{\circ} \mathrm{C}$ mit einer Abweichung von $+2,3^{\circ} \mathrm{C}$ sowie gleichzeitigem Trockenheitsrekord seit 1852 bei einem Niederschlag von $-42 \%$ [7]. Von 1993 bis 2017 sind die Jahresmitteltemperaturen der Tageshöchsttemperatur (THTjm), Tagesmitteltemperatur (TMTjm) und Tagestiefsttemperatur (TTTjm) in Linz progressiv gestiegen. 2018 lagen sie über dem erwarteten Trend, während der Niederschlag über 25 Jahre nicht signifikant zurückging, 2018 aber extrem niedrig war (Abb. 1a). Bei Zunahme von Hitzewellen steigt die Gefahr von Dürre in einem an sich schon niederschlagsarmen Gebiet [8]. Der Klimawandel spiegelt sich für Linz in der Zunahme der Tage $>20^{\circ} \mathrm{C}$ $\left(\mathrm{R}^{2}=0,26 ; \mathrm{p}=0,009\right)$ und $>25^{\circ} \mathrm{C}\left(\mathrm{R}^{2}=0,18 ; \mathrm{p}=\right.$ 0,035) im Zeitraum 1993 bis 2017 wider. Parallel

\begin{tabular}{|c|c|}
\hline \multicolumn{2}{|l|}{ Abkürzungen } \\
\hline Frühblüher & Bäume und Sträucher - bei allergenen Pflanzen: Hasel, Erle, Birke \\
\hline MTF & $\begin{array}{l}\text { mittlerer Tagesfrost }=1 \int_{24} \text { Stundentemperaturen }<0^{\circ} \mathrm{C} / 24 \text { Stunden; Frost definiert als } \\
\text { Temperatur }<0^{\circ} \mathrm{C} \text { mit Gefrierpunkt des Wassers als pflanzenphysiologischer Grenztemperatur }\end{array}$ \\
\hline MTF $_{\text {kumul }}$ & mittlerer Tagesfrost kumuliert ab 1. Januar jeden Jahres \\
\hline MTW & mittlere Tageswärme $=1 \int_{24}$ Stundentemperaturen $>0^{\circ} \mathrm{C} / 24$ Stunden \\
\hline MTW kumul $_{1}$ & mittlere Tageswärme kumuliert ab 1. Januar jeden Jahres \\
\hline NS & Tagesniederschlag in $\mathrm{mm}=\mathrm{L} / \mathrm{m}^{2}$ \\
\hline $\mathrm{NS}_{\text {kumul }}$ & Tagesniederschlag kumuliert ab 1. Januar \\
\hline PF & Pollenflug = Zeit der Pollination = männliche Blüte windblütiger Pflanzen \\
\hline PF-Beginn & $\begin{array}{l}\text { Pollenflugbeginn (PFB): phänologisch definiert als Tag des erstmaligen Erreichens der } \\
\text { PK } 10 \text { Pollen } / \mathrm{m}^{3} \text { (PK10) bei Hasel, Erle und Birke und PK } 5 \text { Pollen } / \mathrm{m}^{3} \text { (PK5) bei Gräsern }\end{array}$ \\
\hline PF-Ende & Pollenflugende analog zu PF-Beginn definiert als letztmaliges Erreichen von PK10 bzw. PK5 \\
\hline PK & mittlere Pollenkonzentration eines Tages als Pollen $/ \mathrm{m}^{3} \mathrm{Luft}$ \\
\hline Pollensaison & PF-Dauer: definiert als Datum des PF-Endes minus PF-Beginns + 1 Tag \\
\hline RF & $\begin{array}{l}\text { relative Feuchte in } \%=\text { effektive Feuchte/Sättigungsfeuchte } \times 100=\text { effektiver Dampfdruck/ } \\
\text { Sättigungsdampfdruck der Luft } \times 100\end{array}$ \\
\hline $\mathrm{RF}_{\text {kumul }}$ & $\begin{array}{l}\text { RF kumuliert ab 1. Januar: wenn hoch, Ausdruck von längeren feuchten; wenn niedrig, von } \\
\text { trockenen Phasen (Dürre) }\end{array}$ \\
\hline Sommerblüher & Kräuter + Stauden - Vertreter bei Allergophyten: Gräser \\
\hline SPIn & saisonales Pollenintegral = Summe der saisonalen mittleren Pollenkonzentrationen \\
\hline THT & Tageshöchsttemperatur \\
\hline TMT & Tagesmitteltemperatur $=1 \int_{24}$ Stundentemperaturen $/ 24$ Stunden \\
\hline TTT & Tagestiefsttemperatur \\
\hline Wärme & definiert als Temperatur $>0^{\circ} \mathrm{C}$ mit Gefrierpunkt als vegetativer Grenze \\
\hline
\end{tabular}




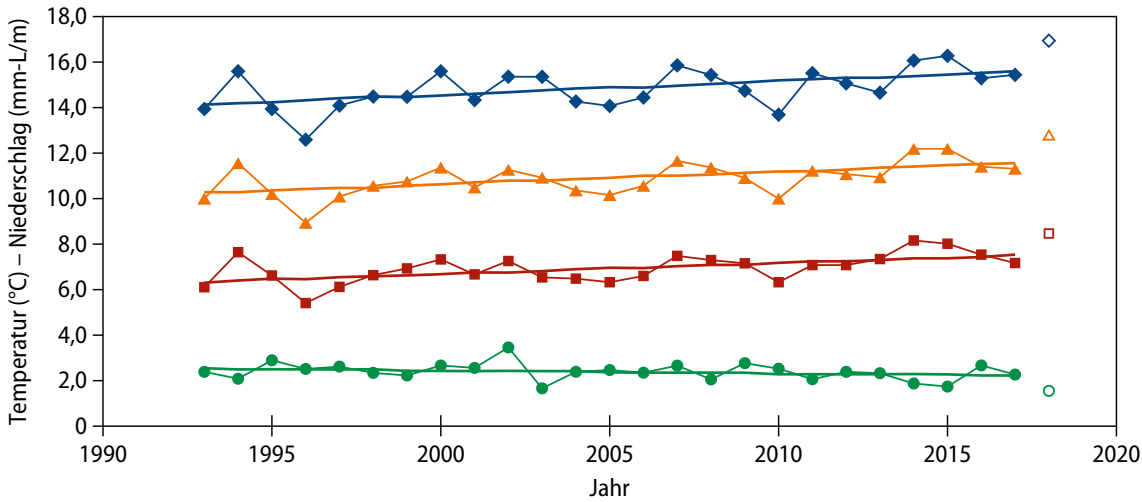

$\mathrm{THT}_{93-18}=0,0722 \mathrm{x}-129,9$

$R^{2}=0,3455 ; p=0,0016$

$\mathrm{THT}_{\mathrm{T} 93-17}=0,0605 \mathrm{x}-106,39$

$R^{2}=0,2712 ; p=0,0076$

$\mathrm{TMT}=0,0549 \mathrm{x}-99,18$

$R^{2}=0,3058 ; p=0,0042$

$\rightarrow$ THTjm

$\rightarrow$ TTTjm

$\sim$ TMTjm

$\diamond$ THTjm2018

TTTjm2018

$\triangle \quad$ TMTjm2018

$\longrightarrow$ NS/Tag

- NS/Tag

TTाjm $=0,049 x-91,347$

$R^{2}=0,3233 ; p=0,0030$

NS $=-0,0129 x+28,275$

$R^{2}=0,0667 ; p=n . s$.

THTjm, Jahresmittelwerte Tageshöchsttemperatur: TMTjm, Jahresmittelwerte Tagesmitteltemperatur; TTTjm, Jahresmittelwerte Tagestiefsttemperatur: NS/Tag, Niederschlag-Tagesmittel; jm, Jahresmittelwerte; NS, Niederschlag; Während es beim Niederschlag keine signifikante Abnahme gab, nahmen alle Temperaturen signifikant zwischen $1,53^{\circ} \mathrm{C}$ und $1,81^{\circ} \mathrm{C}$ u.

Abb. 1a: Jahresmittelwerte von Tageshöchst-, Tagesmittel- und Tagestiefsttemperaturen sowie Niederschlags-Tagesmittel in Linz - 2018 vs. 1993 bis 2017

b

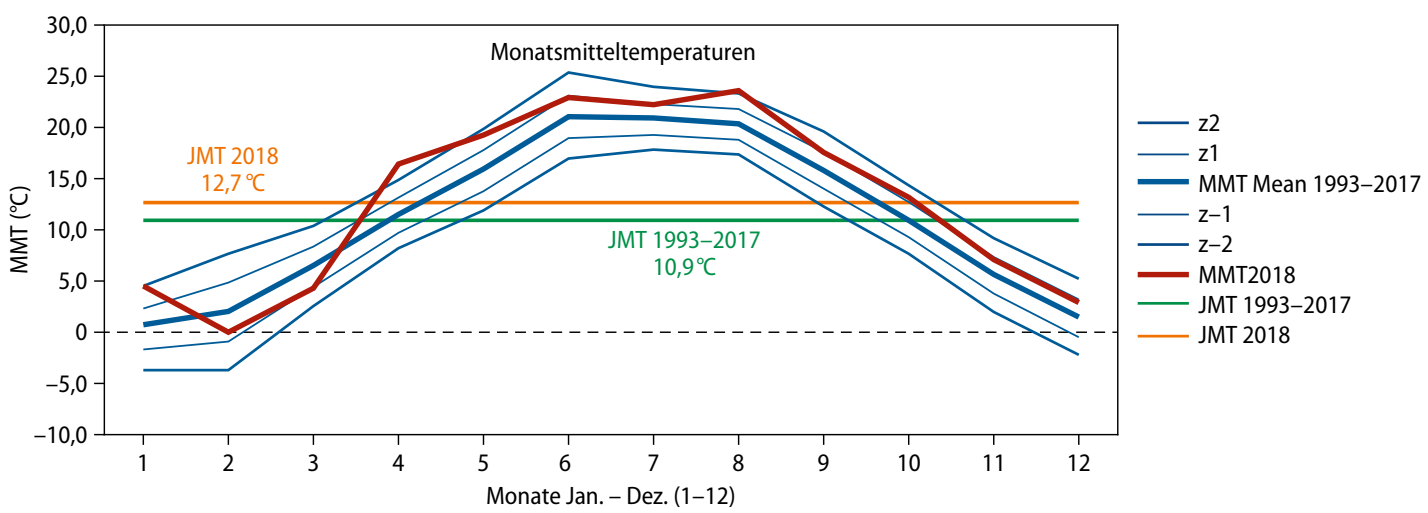

JMT, Jahresmitteltemperatur; MMT, Monatsmitteltemperatur; 2018 wurden über 10 Monate hinweg überdurchschnittliche Monatswerte gemessen (,Wärmejahr"). Februar und März fallen wegen unterdurchschnittlicher Temperaturen durch Frost auf. Entsprechend liegt 2018 ein Frost-Hitze-, das heißt ein Hybridjahr vor, mit unterschiedlichen Einflüssen auf die Frühblüher (Hasel, Erle, Birke) und Sommerblüher (Gräser).

Abb. 1b: Jahresmittel- und Monatsmitteltemperaturen in Linz - 2018 vs. 1993 bis 2017

nahm der Frost bis 2018 signifikant ab $\left(\mathrm{R}^{2}=0,16\right.$, $p=0,027)$. Der Januar 2018 war überdurchschnittlich warm (Monatsmittel: $4,5^{\circ} \mathrm{C}$ ) und gleichzeitig der feuchteste der letzten 26 Jahre (Monatsmittel: $97 \mathrm{~mm}$ ). Nach Kälteeinbrüchen im Februar und März setzte eine Erwärmung ein, die im April zu den höchsten Monatsmitteltemperaturen des letzten Vierteljahrhunderts führten. Dies war gepaart mit einer anhaltenden Trockenheit (relative Feuchte [RF] April: 51,8\% - siehe auch Abb. 8a und 8b). 2018 lag die Temperatur in zehn Monaten über dem Durchschnitt von 1993 bis 2017. Zwei Monate waren unterdurchschnittlich kalt (Abb. 1b).

Die sichtbarste Langzeitfolge der Klimaerwärmung ist ein „früher Frühling“ $[9,10]$. Nach Keenan et al. [11] zieht dieser ein frühes Vegetationsende nach sich. Es steigt zwar durch den verstärkten $\mathrm{CO}_{2}$ Ausstoß die pflanzliche Biomasse in der Nördlichen Biosphäre [12], der Effekt wird aber durch die Häufung saisonaler Wasserdefizite im Sommer und Herbst wettgemacht [13]. Klinisch-palynologisch definieren Blühbeginn, -dauer und -intensität die Pollensaison [14]. Für Blühprognosen des Österreichischen Pollenwarndienstes wurden die Tageshöchsttemperaturen (THT) $[15,16,17]$ und aufsummierten Tagesmitteltemperaturen $\left(\mathrm{TMT}_{\text {cumul }}\right)$ [18] und als Stichtage der 1. Dezember des Vorjahres oder der 1. Januar desselben Jahres verwendet. Begründet wird dies damit, dass (a) mitteleuropäische Bäume und Sträucher einen Kältereiz als Freigabe- 
signal für die temperaturabhängige Entwicklung der Blüte benötigen $[19,20]$ und (b) Temperatursummen aus phänologischer Sicht bei Hasel, Erle und Birke die primäre Einflussgröße ist [21]. Bei der Hasel erfolgen Blattschub und Wachstum nach, bei der Birke parallel zur Blüte. Bei den allergenen Sommerblühern (Gräsern und Kräutern) gehen Pflanzenwachstum und Differenzierung der Blühperiode voraus. Für letztere spielen optimale Start-up-Bedingungen in Form von Temperatur, Niederschlag und RF eine wichtige Rolle [22].

\section{Untersuchungsgebiet}

Linz, die Hauptstadt von Oberösterreich, liegt $260 \mathrm{~m}$ über Adria und ist eine Industriestadt mit 200.000 Einwohnern und weiteren 300.000 in den umliegenden Gemeinden. Die Stadt erstreckt sich über $96 \mathrm{~km}^{2}$ und liegt an der Donau in einem Becken, das von West-Nordwest-Südost (W-NW-SO) vom Kristallin des Böhmischen Massivs mit Höhen bis $930 \mathrm{~m}$ begrenzt wird und nach S-SW gegen das Alpenvorland hin offen ist. Seine Flächen verteilen sich laut Magistrat Linz auf:

_34,8\% Bauland inklusive Industrie

_ 11,6\% Verkehrsflächen

-6,4\% Gewässer sowie

— 47,3\% Wald und Grünland

Hauptpollenquellen sind einerseits die Auwälder der Donau und ihrer Zuflüsse (mit Erlen: Alnus glutinosa und $A$. incana) und der pollenemittierende Grüngürtel um die Stadt (mit Birke = Betula pendula, Hasel $=$ Corylus avellana, Gräsern $=$ Poaceae $)$.

\section{Methoden}

Für die Pollenmonitoring-Station Linz (ATLINZ, N48,303179/O14,305636 ${ }^{\circ}$, Beginn 1985) des Oberösterreichischen Pollenwarndienstes [23] wird eine Burkard-Pollenfalle verwendet, die 20 m über Grund auf einem Dach des Kepler Universitätsklinikums, Med-Campus III (früher AKh Linz) montiert ist. Die Pollenzählung erfolgt standardisiert [24] mittels Lichtmikroskop seit 1987 durch den gleichen Analytiker. Die mittlere Pollenkonzentration eines Tages pro $\mathrm{m}^{3} \mathrm{Luft}(\mathrm{PK})$ wird in die EAN(European Aeroallergen Network)-Datenbank, in Kooperation mit dem Österreichischen Pollenwarndienst in Wien, eingespeist. Von dort wurde sie für die vorliegende Arbeit abgerufen. Saisonal aufsummierte PK werden als saisonales Pollenintegral [25], kurz SPIn, bezeichnet. Durchgehende Messreihen von Erle, Hasel, Birke und Gräser liegen seit 1993 vor. Jahre mit saisonalen Datenlücken $>6$ Tage wurden nicht berücksichtigt.

Beginn und Ende des Pollenflugs (PF) werden phänologisch als erst- und letztmaliges Erreichen von 10 PK für Erle, Hasel und Birke sowie 5 PK für Gräser definiert. Die Zeit dazwischen gilt als PF-
Dauer. Wegen der im Studienzeitraum enthaltenen sechs Schaltjahre wurde Jahrestagen gegenüber dem Kalenderdatum der Vorzug gegeben.

Für den meteorologischen Datensatz von 1993 bis 2018 wurden Stunden- und Tageswerte für Temperatur $\left({ }^{\circ} \mathrm{C}\right)$, Niederschlag $\left(\mathrm{mm}=\mathrm{L} / \mathrm{m}^{2}\right)$ und relative Feuchte (in \%) der Station Linz-Stadt (M1) der Zentralanstalt für Meteorologie und Geodynamik (ZAMG Wien-Salzburg), ein Kilometer von der Pollenfalle entfernt, herangezogen. Aus den Stundenund Tageswerten leiten sich die Tageshöchsttemperaturen (THT), Tagesmitteltemperaturen (TMT) und Tagestiefsttemperaturen (TTT) ab. Davon unterschieden wurde die mittlere Tageswärme (MTW $={ }^{1} \int_{24}$ Stundentemperaturen $>0{ }^{\circ} \mathrm{C} / 24$ Stunden; Wärme definiert als Temperatur $>0{ }^{\circ} \mathrm{C}$ als pflanzenphysiologische Grenztemperatur).

Für den meteorologisch getriebenen PF-Beginn wurden die kumulative mittlere Tageswärme $\left(\mathrm{MTW}_{\text {kumul }}\right.$ ab 1. Januar = Summe der mittleren Tageswärme) sowie eine distinkte THT-Schwelle benutzt. Davon abgegrenzt wurde analog der Einfluss von Frost $\left(\mathrm{MTF}_{\mathrm{kumul}}=\right.$ Summe des mittleren Tagesfrosts $<0{ }^{\circ} \mathrm{C}$ ). Für $\mathrm{MTW}_{\text {kumul }}$ und $\mathrm{MTF}_{\text {kumul }}$ wurden von 1993 bis 1999 Halbstundentemperaturen der Messstation M2 am 24er-Turm der Stadt Linz (Oberösterreichisches Luftmessnetz, OÖ Luftmessnetz), circa 2,2 Kilometer von der Pollenfalle entfernt, verwendet; ab dem Jahr 2000 Stundentemperaturen der ZAMG (M1).

Für die Abbildungen, Tabellenkalkulationen und Regressionen wurden die Programme Microsoft Excel 2003 und 2010 sowie SigmaPlot von Systat Software Inc. (zusammen mit den in den Programmen implementierten Analysefunktionen) verwendet.

\section{Ergebnisse \\ Erle (Alnus) und Hasel (Corylus)}

Pollenflug (PF)-Beginn-, -Verlauf und -Dauer

Frost verzögert den Blühbeginn der Erle signifikant.

Die größten Verzögerungen traten 2006 (Frostmaximum, Tag 86), 2005 und 2003 auf (Abb. 2). Die hohe Varianz des PF-Beginns (Tab. 1) erklärt sich aus unterschiedlich intensiven Frostphasen.

Abb. 3 zeigt die hoch signifikante Verzögerung des Erreichens des erforderlichen $\mathrm{MTW}_{\text {kumul- }}$ und $\mathrm{THT}_{\text {kumul }}$-Niveaus durch Frost bei der Blüte beziehungsweise dem PF-Beginn der Hasel. Bedingt durch den warmen, beinahe frostfreien Januar 2018, erfolgte der PF von Erle und Hasel zum frühesten Zeitpunkt am 31. Januar. Diesem Tag entsprechen eine $\mathrm{MTW}_{\text {kumul }}$ von $126,4^{\circ} \mathrm{C}$ und eine maximale THT ein bis fünf Tage vor PF-Beginn von $11,2^{\circ} \mathrm{C}$ (Tab. 2). $\mathrm{MTW}_{\text {kumul }}$ als präsaisonale Entwicklungstemperatur muss bis zum PF-Beginn relativ konstant im Mittel $103,8^{\circ} \mathrm{C}$ (Median $99,9^{\circ} \mathrm{C}$ ) erreicht haben, ähnlich $\mathrm{THT}_{\text {kumul }}$ als Blüherfordernis mittlere $175,1^{\circ} \mathrm{C}$. 


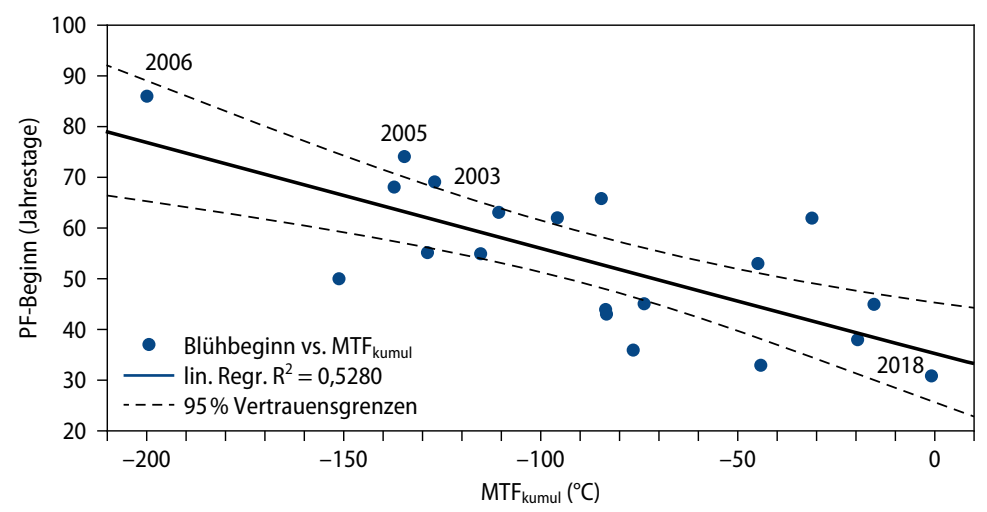

MTF $_{\text {kumul }}$ mittlerer Tagesfrost kumuliert ab 1. Januar jeden Jahres; PF-Beginn, Pollenflugbeginn; Die lineare Korrelation $\left(R^{2}=0,528 ; p=0,002\right)$ zeigt den Einfluss von Frost $\left(M T F_{\text {kumul }}\right)$ auf die Verzögerung des Blühbeginns 1993 bis 2018 (19 Saisons). Die Jahre 2006 und 2005 zeigen die größten Verzögerungen gegenüber 2018; 2006 liegt ein Frostmaximum vor. Die 95 \%-Vertrauensgrenzen sind eingetragen.

Abb. 2: Blühbeginn von Erle (Alnus) vs. Frost [MTF $\left.{ }_{\text {kumul }}\right]$

Jahre mit frühem Blühbeginn tendieren zu einer längeren Pollensaison. Da die PF-Dauer der Hasel signifikant $\left(R^{2}=0,72 ; p<0,001\right)$ mit der Zunahme der Tageswärme korreliert, ist der PF in warmen Jahren verlängert, ohne mit einem erhöhten SPIn einherzugehen.

\section{Einflüsse durch Frost auf Blüte und}

Pollenproduktion von Erle und Hasel

Minimale Frostepisoden mit $\mathrm{MTF}_{\text {kumul }}$ knapp unter $0{ }^{\circ} \mathrm{C}$ gingen dem Blühbeginn der Hasel und Erle 2018 voraus (Abb. 4). Vom 24.2. bis 4.3.2018 kam es zu einem nachhaltigen Kälteeinbruch, der zu einer Unterbrechung der Haselblüte führte. Die Erlenblüte zeigte einen mit der Hasel korrespondierenden frühen Blühbeginn mit niedrigen $\mathrm{PK}$, dafür eine verspätete Hauptphase. Diese bestand aus zwei mar- kanten Pollengipfeln, die ein neuerlicher Kälteeinbruch zwischen 18.3. und 22.3. beendete. Die Erle scheint durch Frost vor und nach Blühbeginn nicht unter Frostschäden zu leiden. Die tiefsten MTF 2018 von $-4,6$ bis $-9,1^{\circ} \mathrm{C}$ lagen zwischen Tag 56 bis 61 , das heißt fünf bis acht Tage vor dem ersten großen Pollengipfel. Betrachtet man das Jahr 2018 isoliert, könnte man auf einen gepulsten, zeit- und möglicherweise dosisabhängigen Kälteeinfluss schließen. Betrachtet man aber das Jahr im Kontext aller Jahre von 1993 bis 2018, so sind die SPIn linear positiv mit zunehmendem Frost bis Pollenflugende $\left(R^{2}=0,196\right.$; $\mathrm{p}=0,0575)$ sowie negativ mit Wärme $\left(\mathrm{MTW}_{\mathrm{kumul}}\right.$ $\left.\mathrm{R}^{2}=0,276, \mathrm{p}=0,021\right)$ korreliert. Dies weist darauf hin, dass der positive Einfluss von Minus- sowie kühlen Temperaturen auf die Steigerung der SPIn der Erle ein zeitlich nicht genau begrenzter Effekt ist. Im Gegensatz zur Erle spielt präsaisonaler Frost bei der Hasel keine Rolle für die SPIn $\left(\mathrm{R}^{2}<0,1\right)$. Wärme treibt die SPIn von Hasel bis zum Erreichen eines Sättigungswertes der $\mathrm{MTW}_{\text {kumul }}$ von circa $500{ }^{\circ} \mathrm{C}$.

\section{Birke (Betula)}

Pollenflugbeginn und -dauer

Der PF-Beginn von 1993 bis 2018 schwankte auffällig gering um den Median 91,5 und arithmetischen Mittelwert (Mean) \pm 1 Standardabweichung (SD) $93,0 \pm 7,2$ Tagen (Tab. 1). Beim Blühbeginn am 2.4.2018 bestanden eine Entwicklungstemperatur $\mathrm{MTW}_{\mathrm{kumul}}$ von $314,2^{\circ} \mathrm{C}$ und eine Öffnungstemperatur von THT 13,7 bis $14,5^{\circ} \mathrm{C}$. In Abb. 5 ist exemplarisch für die Birke dargestellt, wie der Blühbeginnkorridor durch Interaktion von drei Faktoren bestimmt wird: artspezifischer Blühbeginn, kumulierter MTW und THT-Schwelle. Für alle Saisons verschiebt Frost den Birken-PF nur marginal

Tab. 1: Pollenflugbeginn von Hasel (Corylus), Erle (Alnus), Birke (Betula) und Gräsern (Poaceae)

\begin{tabular}{|l|l|l|l|l|l|}
\hline Pollenflugbeginn (PFB) & Hasel PK10 Tag & Erle PK10 Tag & Birke PK10 Tag & Gräser PK5 Tag \\
\hline Vorverlagerung-Trend & keiner $p=0,34$ & keiner $p=0,88$ & keiner $p=0,59$ & Tendenz $p=0,062$ \\
\hline Mittelwert & 55,4 & 51,7 & 93,0 & 118,6 \\
\hline Standardfehler & 3,43 & 2,90 & 1,79 & 2,40 \\
\hline Median & 58,5 & 50,0 & 91,5 & 117,0 \\
\hline Standardabweichung & 15,35 & 12,64 & 7,16 & 8,98 \\
\hline Minimum & 31 & 31 & 81 & 107 \\
\hline Maximum & 86 & 74 & 109 & 136 \\
\hline Saisonen & 20 & 19 & 16 & 14 \\
\hline SD\% Mean & 27,7 & 24,4 & 7,7 & 7,6 \\
\hline
\end{tabular}

Pollenflugbeginn-Korridor $=$ Streuung des Pollenflugbeginns 

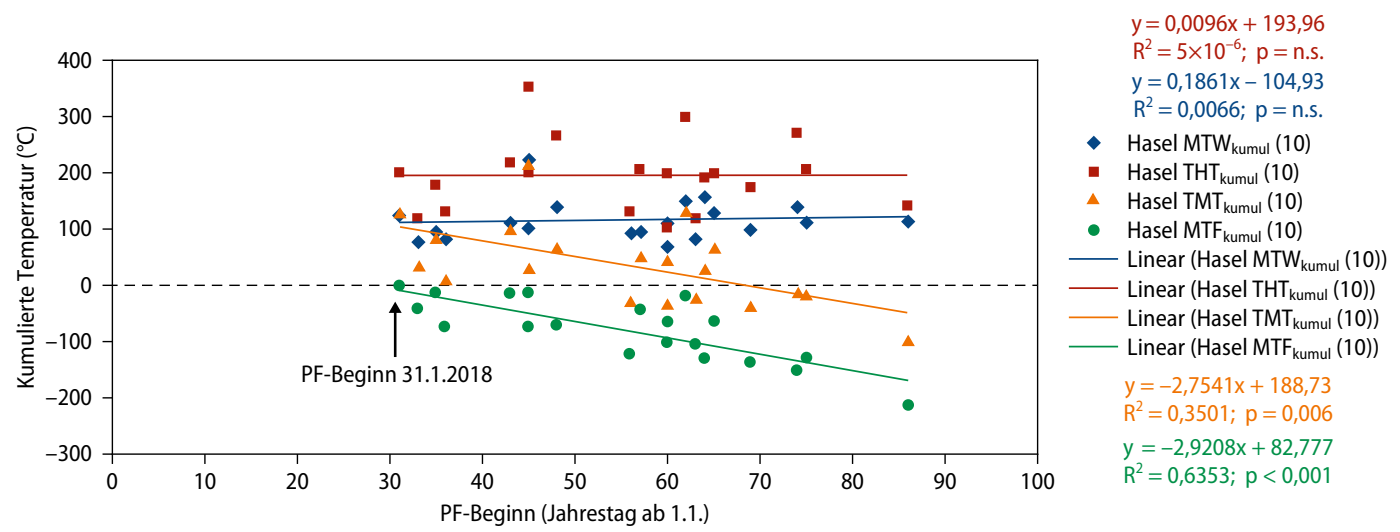

Die kumulierte mittlere Tageswärme (MTW kumul muss als präsaisonale Entwicklungstemperatur bei Blühbeginn im Mittel $104,6^{\circ} \mathrm{C}$ erreicht haben. Ähnlich

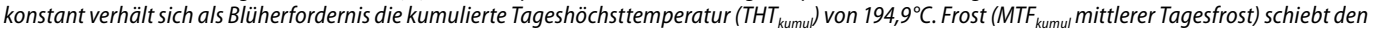

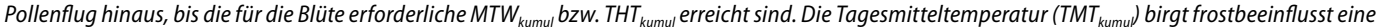
ähnliche Information wie MTFkumul, ist mit $R^{2}=0,35$ aber geringer korreliert. Der PF-Beginn der Hasel 2018 am Tag 31 war der früheste in $26 \mathrm{Jahren}$, bedingt durch minimalen präsaisonalen Januarfrost von nur MTF $_{\text {kumul }}-0,9^{\circ} \mathrm{C}$.

Abb. 3: Hasel (Corylus) PK 10- kumulierte Temperaturen am Pollenflugbeginn von 1993 bis 2018 (20 Saisons) [MTW $\left.\mathrm{Mumul}_{\text {, }} \mathrm{TMT}_{\text {kumul, }} \mathrm{THT}_{\text {kumul, }} \mathrm{MTF}_{\text {kumul }}\right]$

$\left(\mathrm{R}^{2}=0,22 ; \mathrm{p}=0,066\right)$. Die Blüherfordernis bleibt relativ konstant bei $\mathrm{MTW}_{\text {kumul }}$ von 336,1 $\pm 55,8$ (Mean $\pm 1 \mathrm{SD}$, Median $335,4^{\circ} \mathrm{C}$ ). Die THT bei PF-Beginn betrug bei 16 Saisons ein bis fünf Tage vor PF-Beginn $17,5 \pm 3,7^{\circ} \mathrm{C}$ (Tab. 2). Analoge PF-Beginnkorridore sind für Hasel und Erle konstruierbar.
Die Dauer der Birkenpollensaisons steigt signifikant mit dem intrasaisonalen Wärmeangebot $\left(\mathrm{R}^{2}=\right.$ 0,7953; $\mathrm{p}<0,001$ für $\left.\mathrm{MTW}_{\mathrm{kumul}}\right)$. Der Einfluss von Niederschlag im Sinne eines humiden Anspruchs der Birke ist vernachlässigbar $\left(\mathrm{NS}_{\text {kumul }} \mathrm{R}^{2}=0,14 ; \mathrm{p}=\right.$ 0,15). Eine Sonderstellung nimmt das Jahr 2018 ein,

\section{Tab. 2: Zusammenhang von Tageshöchsttemperatur und Pollenflugbeginn von Hasel (Corylus), Erle (Alnus), Birke (Betula) und Gräsern (Poaceae)}

\section{Tageshöchsttemperatur}

\begin{tabular}{|c|c|c|c|c|c|}
\hline 1993-2018 & Saisons & THT-Bereich & Median & Mean \pm 1 SD & Bereich \\
\hline \multirow{2}{*}{$\begin{array}{l}\text { Hasel (Corylus) } \\
\text { PK5 }\end{array}$} & \multirow[t]{2}{*}{20} & $\operatorname{Tag} \varnothing$ & 11,10 & $9,95 \pm 5,02$ & $1,5-18,1$ \\
\hline & & Tag -1 bis -5 & 11,05 & $10,79 \pm 3,22$ & $4,4-16,9$ \\
\hline \multirow{2}{*}{$\begin{array}{l}\text { Hasel (Corylus) } \\
\text { PK10 }\end{array}$} & \multirow[t]{2}{*}{20} & $\operatorname{Tag} \varnothing$ & 11,65 & $11,30 \pm 4,16$ & $2,7-18,1$ \\
\hline & & Tag -1 bis -5 & 11,70 & $11,55 \pm 2,88$ & $5,1-16,9$ \\
\hline \multirow{2}{*}{$\begin{array}{l}\text { Erle (Alnus) } \\
\text { PK5 }\end{array}$} & \multirow[t]{2}{*}{19} & $\operatorname{Tag} \varnothing$ & 9,50 & $9,64 \pm 4,44$ & $1,5-16,2$ \\
\hline & & Tag -1 bis -5 & 10,90 & $10,57 \pm 3,47$ & $4,1-16,9$ \\
\hline \multirow{2}{*}{$\begin{array}{l}\text { Erle (Alnus) } \\
\text { PK10 }\end{array}$} & \multirow[t]{2}{*}{19} & $\operatorname{Tag} \varnothing$ & 10,30 & $10,17 \pm 4,15$ & $1,5-16,6$ \\
\hline & & Tag -1 bis -5 & 10,30 & $10,63 \pm 3,51$ & $4,5-16,9$ \\
\hline \multirow{2}{*}{$\begin{array}{l}\text { Birke (Betula) } \\
\text { PK10 }\end{array}$} & \multirow[t]{2}{*}{16} & Tag $\varnothing$ & 16,65 & $16,91 \pm 3,87$ & $10,5-23,9$ \\
\hline & & Tag -1 bis -5 & 16,45 & $17,51 \pm 3,72$ & $9,7-23,1$ \\
\hline \multirow{2}{*}{$\begin{array}{l}\text { Birke (Betula) } \\
\text { PK100 }\end{array}$} & \multirow[t]{2}{*}{16} & $\operatorname{Tag} \varnothing$ & 16,65 & $15,51 \pm 5,64$ & $4,6-23,9$ \\
\hline & & Tag -1 bis -5 & 16,65 & $17,54 \pm 3,89$ & $10,6-23,1$ \\
\hline \multirow{2}{*}{$\begin{array}{l}\text { Gräser (Poaceae) } \\
\text { PK5 }\end{array}$} & \multirow[t]{2}{*}{14} & $\operatorname{Tag} \varnothing$ & 20,05 & $20,00 \pm 4,06$ & $13,2-29,7$ \\
\hline & & Tag -1 bis -5 & 21,35 & $21,10 \pm 3,16$ & $15,2-26,0$ \\
\hline \multirow{2}{*}{$\begin{array}{l}\text { Gräser (Poaceae) } \\
\text { PK10 }\end{array}$} & \multirow[t]{2}{*}{14} & $\operatorname{Tag} \varnothing$ & 19,95 & $19,76 \pm 6,66$ & $8,7-30,2$ \\
\hline & & Tag -1 bis -5 & 22,95 & $23,14 \pm 3,50$ & $14,9-29,4$ \\
\hline
\end{tabular}

Pollenflugschwelle: PK5, Pollenkonzentration 5 Pollen/m3; PK10, Pollenkonzentration 10 Pollen/m3; PK100, Pollenkonzentration 100 Pollen/m3; Tageshöchsttemperatur: THT Ø, Tageshöchsttemperatur am Tag des Pollenflugbeginns; THT Tag -1 bis -5; Tageshöchsttemperatur ein bis fünf Tage vor Pollenflugbeginn; SD, "standard deviation" 


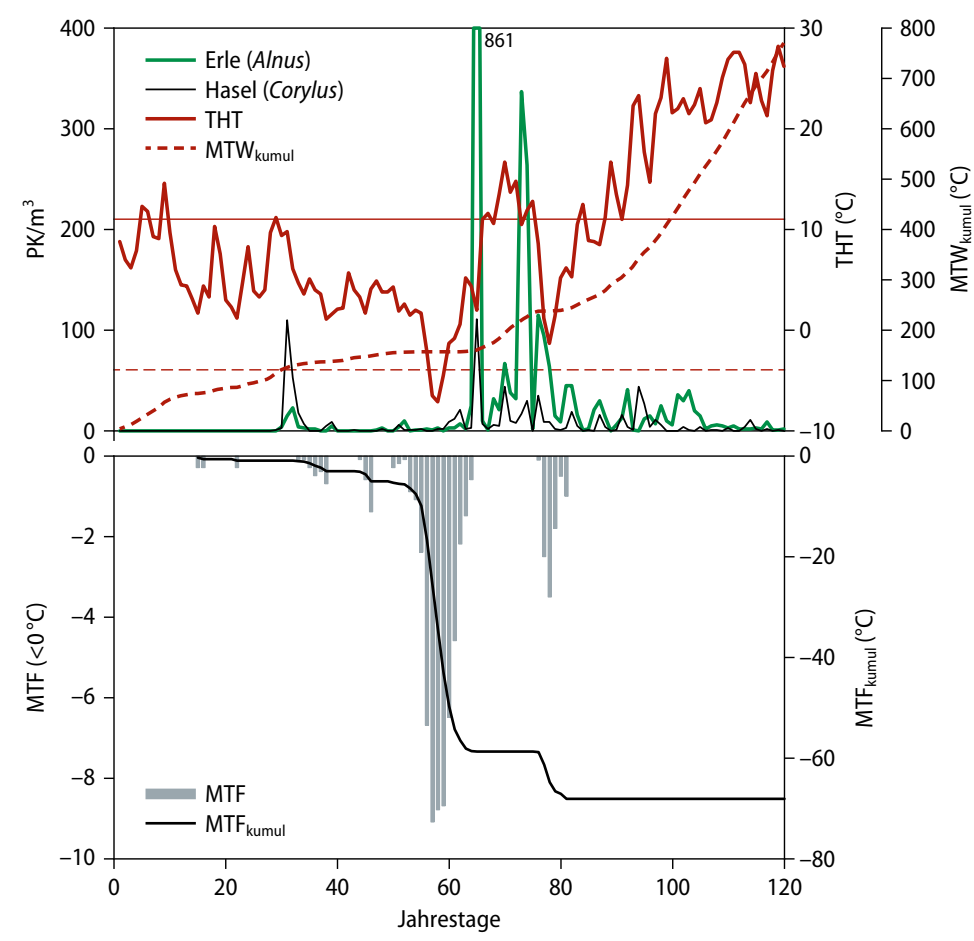

$P K$, Pollenkonzentration (je $\mathrm{m}^{3}$ ); $M_{T} F_{\text {kumul, }}$ mittlerer Tagesfrost; $M T W_{\text {kumul, }}$ kumulierte mittlere Tageswärme; THT, Tageshöchsttemperatur; ${ }^{*}$ Pollenkonzentrationen der Erle vom 31.1. bis 30.4. Schwache Frosteinflüsse $\left(\right.$ MTF $\left._{\text {kumul }}-0,9^{\circ} \mathrm{C}\right)$ im Januar vor Blühbeginn bei Koinzidenz von $\mathrm{MTW}_{\text {kumul }}\left(126,4^{\circ} \mathrm{C}\right.$ - unterbrochene Linie) und der THT-Schwelle $\left(11,2^{\circ} \mathrm{C}\right)$ als Blüherfordernis - analog früher Pollenflugbeginn der Hasel. Frost im Februar und März führte zu einer Blühunterbrechung der Hasel und Verzögerung der Hauptblüte der Erle. Auch die tiefsten Temperaturen zeigten keine Frostschädigung der Erlenblüte, im Gegenteil deuten sie sogar auf eine frostinduzierte Stimulierung der Pollenproduktion hin.

Abb. 4: Einfluss von Wärme und Frost auf die Konzentration von Erlenpollen (Alnus) und Haselpollen (Corylus) 2018 in dem vor allem im April die ausgeprägte Niederschlagsarmut (Dürre) in Kombination mit Hitze die PF-Dauer verkürzte (Abb. 6a).

Der Einfluss niederer präsaisonaler Temperaturen auf die Pollenproduktion

Die Birke wies 2003 das höchste SPIn auf. Auch die Jahre 2004, 2005 und 2006 liegen über dem 26-Jahre-Trend [18] ähnlich wie 2018. Die tiefsten MTF von $-4,1$ bis $-5,2{ }^{\circ} \mathrm{C}$ traten 2003 zwischen Tag 45 bis 49 auf, das heißt 57 bis 65 Tage vor dem ersten großen Pollengipfel der Birke. Es liegt kein direkter zeitlicher Bezug von SPIn zu Frost vor. Wie bei der Erle sind aber die SPIn der Birke linear negativ $\left(\mathrm{R}^{2}=0,3259 ; \mathrm{p}=0,0209\right)$ mit präsaisonaler $\mathrm{MTW}_{\text {kumul }}$ korreliert, das heißt, kühlere Temperaturen stimulieren die Pollenproduktion (SPIn) (Abb. 6b).

\section{Gräser (Poaceae)}

Pollenflug (PF)-Beginn und -Dauer

Es zeichnet sich eine Tendenz $\left(R^{2}=0,2614 ; \mathrm{p}=\right.$ $0,062)$ zu einem früheren PF-Beginn ab. Der früheste erfolgte 2018 am 107. Jahrestag. Die PF-Dauer bleibt über die Jahre annähernd konstant $\left(\mathrm{R}^{2}<0,1\right)$. Sie lag 2018 bei 108 Tagen. Die PF-Saisons dauern bei PK5 $106,1 \pm 17,2$ Tage (Mean \pm 1 SD).

Die Gräserpollensaison 2018 war zeitlich gegenüber 1994 bis 2017 vorverlagert, parallel dazu der Haupt-Peak (Abb. 7a). Da die Zahl der Gräserpollen-Peaks zwischen 1994 bis 2017 (30) und 2018 (26) ähnlich ist, dürfte sie primär auf eine gestaf-

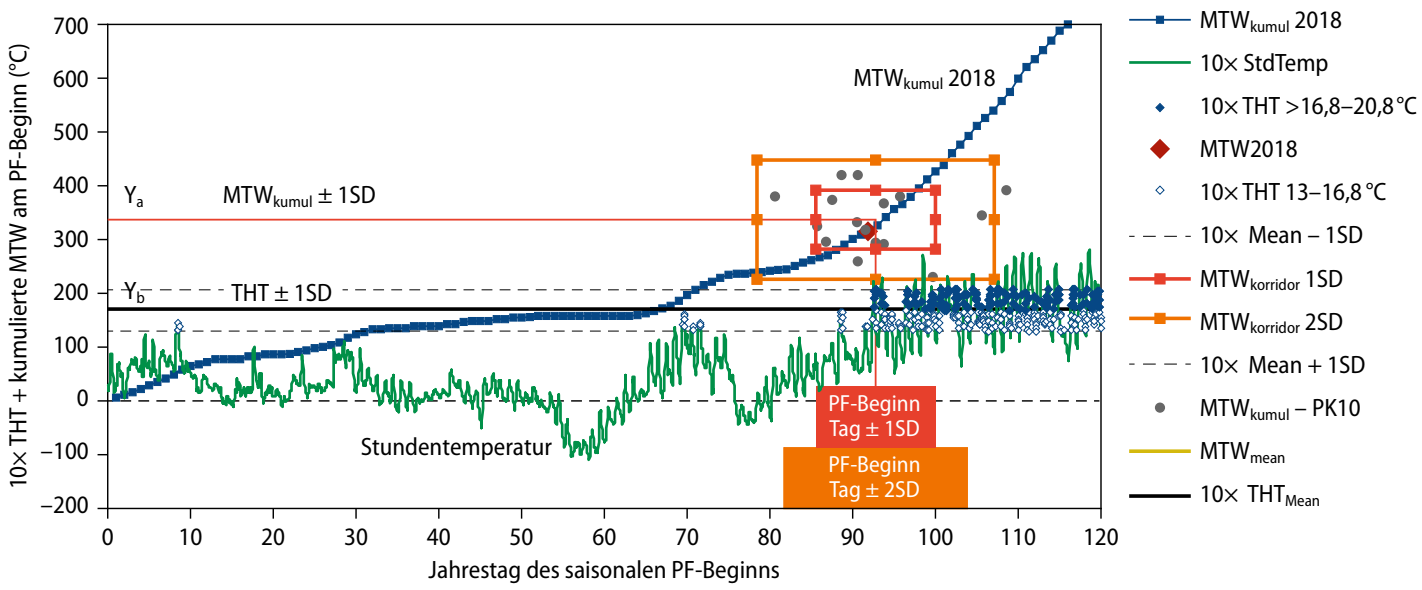

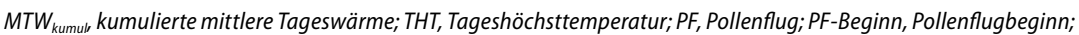
Der Bereich des Pollenflugbeginns zwischen 1993 bis 2017 (15 Saisons) lag bei Tag 93,0 \pm 14,2 (Mean \pm 2 SD; Median 91,5; Bereich 81-109; aufx-Achse). Der Pollenflug beginnt beim Zusammentreffen von kumulierter mittlerer Tageswärme ( $M T W_{\text {kumul }} M \pm 1 S D 336,1 \pm 55,8^{\circ} \mathrm{C}$; aufy-Achse) und Überschreiten einer minimalen Tageshöchsttemperatur (THTminim $>13,6^{\circ} \mathrm{C}$ während der letzten 5 Tage vor PF bzw. $>13,0^{\circ} \mathrm{C}$ am PF-Tag $\emptyset ; y$-Achse $e_{b}$ ) innerhalb des

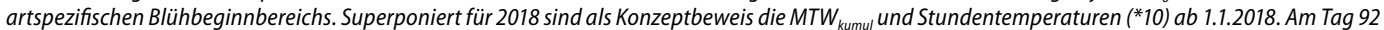
setzte 2018 der Birkenpollenflug bei MTW kumul $^{3} 314,2^{\circ} \mathrm{C}$ (als Entwicklungstemperatur) und koinzidenter $T H T_{\text {minim }}$ (Öffnungstemperatur) von 13,7 bis $14,5^{\circ} \mathrm{C}$ ein. $M_{\text {MTWul }}$ und THT-Schwelle bieten sich für die meteorologische Vorhersage des PF-Beginns an.

Abb. 5: Blühbeginnkorridor für die Birke (Betula): PF-Beginn 2018 am Tag 92 im Korridor der Birkenblüte bei

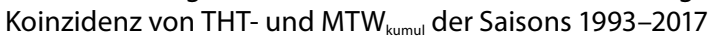


felte beziehungsweise gepulste Blühfolge diverser Gräserarten zurückgehen. Die höheren Pollengipfel in der ersten Hälfte der Saison deuten auf pollenproduktivere Arten hin [26]. Die niedrigeren PK der zweiten Saisonhälfte sind wahrscheinlich auch vom landwirtschaftlichen Grasschnitt beeinflusst.

\section{Einflüsse von Feuchte und Trockenheit auf die Pollenproduktion}

Die präsaisonale kumulierte relative Feuchte $\left(\mathrm{RF}_{\text {kumul }}\right)$ weist eine hoch signifikante Korrelation mit dem PF-Beginn auf $\left(\mathrm{R}^{2}=0,8826 ; \mathrm{p}<0,001\right)$ (Abb. 8a), die deutlich die Einzelgrößen Wärme $\left(R^{2}=0,3173 ; p=\right.$ $0,005)$ und Niederschlag $\left(R^{2}=0,3377 ; p=0,004\right)$ für Gräserwachstum und Blütenanlage [27] übertrifft. RF repräsentiert dabei das Verhältnis von Niederschlag, Bodenfeuchte und Evaporation zur Temperatur (Wärme). Eine niedrige RF steht für Trockenheit beziehungsweise ein Feuchtedefizit. Trockenheit beziehungsweise Dürre führen zu einem frühen PF-Beginn, während Nässe den Blühbeginn verzögert. Wegen der hoch signifikanten Beziehung zwischen $\mathrm{RF}_{\text {kumul }}$ und PF-Beginn bietet sich die relative Feuchte für die Pollenflugvorhersage der Gräser an.

Die höchsten Gräser-SPIn traten in den Jahren 1994, 2001, 2002 und 2004 auf. Über die 26 Jahre nahm die kumulierte RF kontinuierlich ab (Abb. 8b), was auf eine zunehmende Trockenheit hinweist. Bei linearer Regression nahm die Pollenproduktion von 1994 bis 2018 ab sowohl was die Gesamtzahl (SPIn $\left.\mathrm{R}^{2}=0,36 ; \mathrm{p}=0,024\right)$ als auch die PK der Haupt-Pollen-Peaks $\left(R^{2}=0,32 ; p=0,035\right)$ betrifft. Durch die größeren Datenlücken ergibt sich jedoch eine Unsicherheit trotz Ausdehnung von 14 PF-Saisons über 25 Jahre. Die polynome Regression zeigt bei einem $R^{2}=0,39$ einen biphasischen Verlauf, mit einer Stabilisierung auf niedrigerem Niveau in den Jahren 2005 bis 2018 (Abb. 7b).

\section{Diskussion}

In Linz nahm über 26 Jahre die Temperatur zu und zwar im Sommer- mehr als im Winterhalbjahr. Während die Minustemperaturen pro Jahr kaum abnahmen, nahm die MTW pro Jahr um $0,056{ }^{\circ} \mathrm{C}$ $\mathrm{zu}\left(\mathrm{R}^{2}=0,4151 ; \mathrm{p}<0,001\right)$. Die Korrelation zwischen Wärmezu- und Frostabnahme ist hoch signifikant $\left(R^{2}=0,519 ; p=0,00002\right)$, was für eine parallele Verringerung/Verkürzung der winterlichen Frostzeiten spricht [28]. Dagegen fiel im Beobachtungszeitraum der Niederschlag tendenziell, sodass sich das Klima regional in Richtung warm-trocken entwickelte; ablesbar an der kontinuierlich fallenden mittleren RF. Linz als „urbanes Pflaster“ liegt in einer niederschlagsarmen Region, in der sich meteorologische Grenzsituationen wie Niederschlagsdefizite und urbane Überwärmung früh auswirken. Bei Fortset-

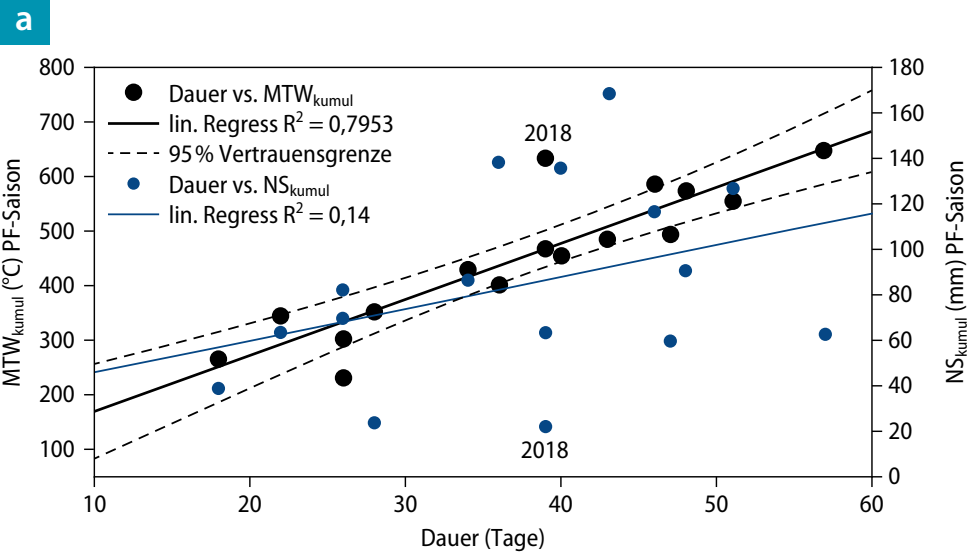

Im Zeitraum von 1993 bis 2018 (16 Saisons) ist ein signifikanter Anstieg der Dauer des Pollenflugs bei steigenden intrasaisonalen Temperaturen ( $M T W_{\text {kumul }}$ mit $R^{2}=0,7953 ; p=0,00018$ ) zu verzeichnen. Dagegen zeigt der Niederschlag $\left(N S_{\text {kumu }}\right)$ nur einen geringfügigen Einfluss $\left(R^{2}=0,14 ; p=0,15\right)$. Ersichtlich ist die Sonderstellung des Trockenjahres 2018: Aus der Kombination von großer Wärme, aber kaum Niederschlag, ergibt sich eine mittlere PF-Dauer.

Abb. 6a: Dauer der Pollensaison von Birke (Betula) 1993 bis 2018 vs. MTW und NS kumuliert

\section{b}

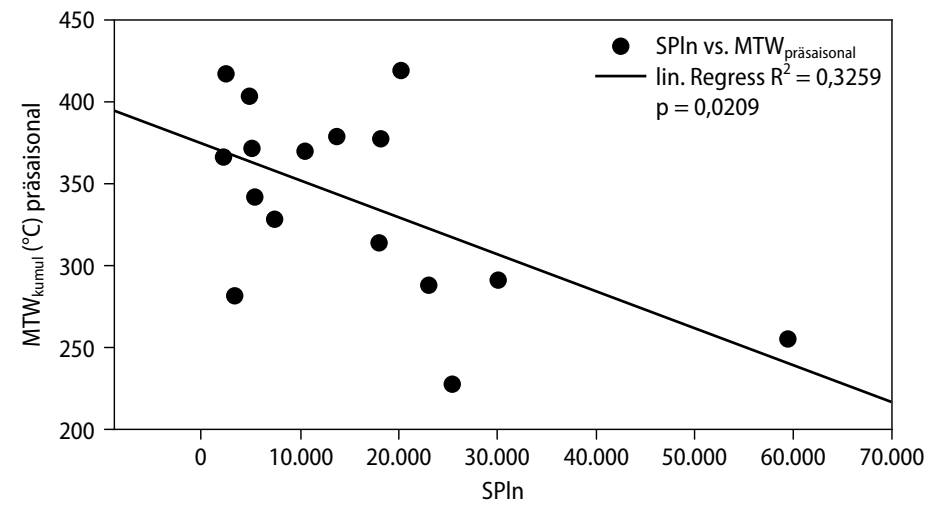

Die saisonalen Pollenintegrale (SPIn) sind linear negativ mit präsaisonaler mittlerer Tageswärme (MTW kumul $\left.R^{2}=0,326 ; p=0,0209\right)$ korreliert. Wärme führt zu niedrigen Pollenkonzentrationen, kühle Temperaturen führen zu einer Erhöhung.

Abb. 6b: Pollenkonzentration von Birke (Betula) 1993 bis 2018 (16 Saisons) vs. präsaisonalen $\mathrm{MTW}_{\text {kumul }}$

zung des langjährigen meteorologischen Trends lässt das außergewöhnliche Jahr 2018 ähnliche Situationen in circa 20 Jahren als "gängig“ antizipieren.

Die Erklärung dafür, dass die regionale Klimaerwärmung und zunehmende Trockenheit über 26 Jahre statistisch keine signifikante (fassbare) Änderung des Pollenflugs von Hasel, Erle, Birke und Gräsern nach sich zog, ist in der variablen Saisonalität zu suchen. Der Grund für diese dürfte in der hohen Varianz der den PF bestimmenden präsaisonalen Wettersituationen liegen. Daraus resultiert, dass über 26 Jahre die kumulierte MTW bis Tag 90 keine signifikante Änderung zeigt $\left(\mathrm{R}^{2}=0,023\right.$; n. s. $)$ im Gegensatz zur Jahreserwärmung $\left(\mathrm{R}^{2}=0,339 ; \mathrm{p}=\right.$ 
a

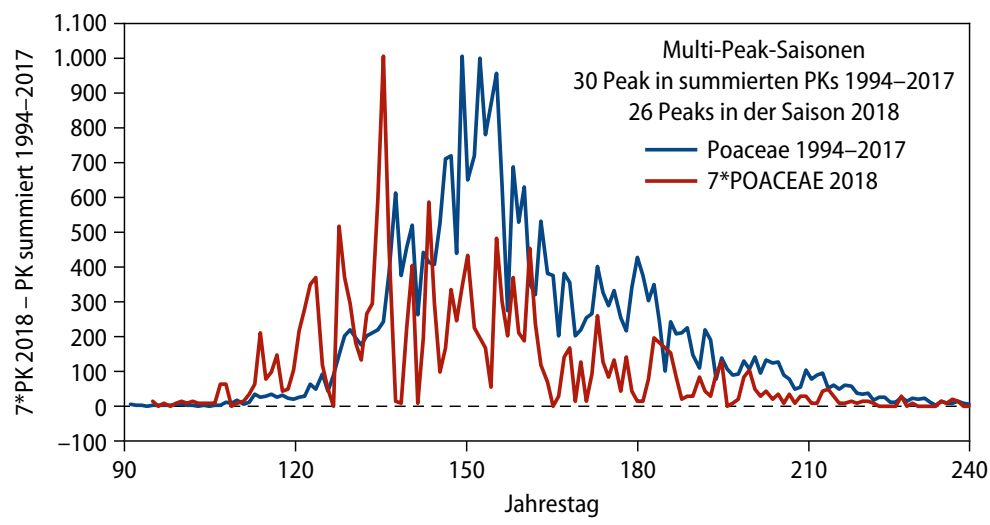

PF, Pollenflug; PK, mittlere Pollenkonzentration eines Tages als Pollen $/ \mathrm{m}^{3} \mathrm{Luft}$; Die PK sind besonders 2018 in der ersten Hälfte höher als in der zweiten Hälfte. Das Multi-Peak-Profil ist sowohl 2018 als auch in früheren Saisonen nachweisbar, ähnlich die Zahl der Pollen-Peaks, wahrscheinlich primär bedingt durch eine gestaffelte Abfolge der Blüte einer Vielzahl von Gräsern. 2018 dauert der PF gleich lang wie früher, ist aber vorverlagert, ebenso der Haupt-Peak.

Abb. 7a: Gräser (Poaceae) - Pollenflugsaison 2018 vs. Mittel von 13 kongregierten Saisons zwischen 1994 und 2017

b

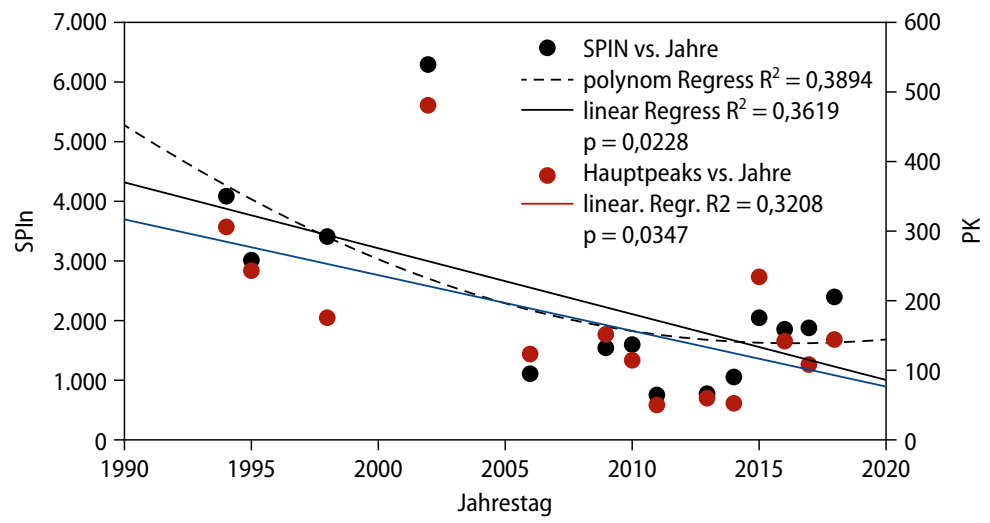

SPIn, saisonales Pollenintegral; PK, mittlere Pollenkonzentration eines Tages als Pollen $/ \mathrm{m}^{3} \mathrm{Luft}$; Bei linearer Regression nehmen beide ab, SPIn mit $R^{2}=0,3619$ und $p=0,02228$, die Haupt-Peaks mit $R^{2}=0,3208$ und $p=0,0347$. Bei polynomer Regression $\left(R^{2}=0,398\right)$ zeichnet sich eine Stabilisierung auf niedrigerem Niveau zwischen 2005 und $2018 a b$.

Abb. 7b: Gräser (Poaceae) - Saisonale Pollenintegrale und Haupt-Peaks 1994 bis 2018 (14 Saisons)

0,0018). Die phänologischen Zusammenhänge und Gesetzlichkeiten hinter dieser Varianz werden diskutiert. Bei Birke und Gräsern ist in Zukunft eher eine Vorverlagerung der PF-Periode zu erwarten; bei den Gräsern scheint sich diese Tendenz bereits abzuzeichnen.

2018 war in Linz bei einer überdurchschnittlichen Temperaturerhöhung in zehn/zwölf Monaten ein Wärmejahr und aufgrund eines Niederschlagsdefizits ebenfalls in zehn/zwölf Monaten ein Trockenjahr. Zusätzliche Kälteperioden im Februar und
März mit Eigengesetzlichkeiten wie höherer Pollenproduktion bei Erle und Birke durch stimulierende Kältereize machen $2018 \mathrm{zu}$ einem ausgesprochenen Hybridjahr.

Bei den frühblühenden Bäumen und Sträuchern Erle, Birke und Hasel beginnt die Anlage und Entwicklung der männlichen Kätzchen schon im Sommer des Vorjahres. Für sie sind im PF-Jahr die Koinzidenz einer distinkten $\mathrm{MTW}_{\text {kumul }}$ als Entwicklungstemperatur zum Erreichen der Blühbereitschaft und die THT als Impuls- beziehungsweise Öffnungstemperatur für den Start der Pollination erforderlich. Das für die Frühblüher exemplarische Modell des Pollenflugbeginnkorridors der Birke illustriert, wie der artspezifische Blühbeginn durch MTW $_{\text {kumul }}$ und eine THTSchwelle zeitlich eingeengt werden. Es umreißt die Kriterien für eine meteorologische PF-BeginnVorhersage. Analoge PF-Beginnkorridore sind für Hasel und Erle konstruierbar, da die Blühbedingungen gleichen Prinzipien folgen. Als dritte meteorologische Determinante des PF-Beginns tritt Frost hinzu. Frost verzögert den Blühbeginn von Hasel und Erle und erklärt dessen hohe Varianz. 2006 erfolgte der PF-Beginn wegen des beobachteten Frostmaximums 55 Tage später als der früheste 2018. Warme beziehungsweise frostarme Winter führen zu einer Blühphasenverschiebung bei Erle und Hasel nach vorne. Die präsaisonalen MTW $_{\text {kumul }}$ und THT-Schwellen blieben bei aller Variabilität in den letzten 26 Jahren auffallend konstant und damit als Haupttreiber des PF-Beginns unverändert.

Die saisonale Pollenproduktion folgt bei Hasel, Erle und Birke anderen Mechanismen als der PFBeginn. Frost führte $2018 \mathrm{zu}$ einer Blühunterbrechung bei Hasel und Erle. Präsaisonaler Frost und kühle Temperaturen stimulierten bei der Erle die Pollenproduktion (SPIn), während die Hasel hierzu der Wärme bedurfte. Die Birke zeigt das Paradoxon, dass sie zwar höhere Temperaturen für die Blühbereitschaft verlangt als Erle und Hasel, aber ebenfalls kühle präsaisonale Temperaturen die SPIn steigern [18]. Aufgrund der nordisch/alpinen beziehungsweise montanen Verbreitungsschwerpunkte von Hängebirke (B. pendula) und Grauerle (A. incana) macht diese Strategie durchaus Sinn, da hauptsächlich Frost im Frühling die Verbreitungsgrenzen bestimmt [29]. Der Mechanismus ist jedoch unbekannt. Die Wärmestimulation der Hasel könnte mit ihrem unterschiedlichen (südosteuropäischen und kleinasiatischen) Herkunftsund Verbreitungsgebiet zusammenhängen. Die Wärme-Frost-Hypothese erklärt Schwankungen der SPIn bei den Frühblühern zwischen Jahren mit Kältestimulierung und Wärmejahren mit frühem Blühbeginn ohne Kältereize. Das Jahr 2003 weist 
die höchsten SPIn von Erle und Birke auf. Solche pollenreichen Jahre („Mastjahre“) lassen sich durch einen Synergieeffekt aus verstärkter präsaisonaler Kältestimulierung der Pollenproduktion und einem stärkeren Knospenansatz im Vorjahr erklären [30]. Steigende Wärme während der Pollensaison verlängert - wie auch bei Hasel und Erle - die PF-Dauer der Birke. Hitze in Kombination mit ausgeprägter Niederschlagsarmut (Dürre) wie 2018 verkürzen diese andererseits. Dies ist für die Intensität der Birkenpollenbelastung in überhitzten urbanen Gebieten von Bedeutung. Im Gegensatz zu den präsaisonalen meteorologischen Bedingungen, die primär die Pollenintensität der Frühblüher steuern, spielt zum Beispiel Wärme während des Pollenflugs statistisch für die SPIn nur eine untergeordnete, modifizierende Rolle.

Die Gräser zeigten 2018 den frühesten PF-Beginn. Der Grund wird in der Trockenheit und auffällig warmen Temperaturen im April gesehen. Die niedrigste RF 2018 reflektiert das Verhältnis beider. Nicht nur, dass über 26 Jahre die kumulierte $\mathrm{RF}$ kontinuierlich gefallen ist, erweist sich $\mathrm{RF}_{\mathrm{kumul}}$ auch als bester Prädiktor für den PF-Beginn der Gräser. Bei multivariater Analyse dominiert $\mathrm{RF}_{\text {kumul }}$ den PF-beginn sosehr, dass die beiden anderen Einflussfaktoren, $\mathrm{MTW}_{\text {kumul }}$ und $\mathrm{NS}_{\text {kumul }}$ unbedeutend werden. Nässe, kombiniert mit Wärme, schiebt dagegen den Blühbeginn nach hinten. Von 1994 bis 2018 nahmen sowohl die Gesamtzahl (SPIn) als auch die Konzentrationen der HauptPollen-Peaks der Gräser linear ab. Bei polynomer Regression zeigt sich ein biphasischer Verlauf mit einer Stabilisierung beider Parameter auf niedrigerem Niveau zwischen 2006 bis 2018. Anstelle längerer Regenperioden, welche die Pollenkonzentrationen allgemein senken, traten 2018 episodische Niederschläge, wie sie für die Klimaerwärmung prognostiziert werden [31]. Diese können das Graswachstum und die Pollenproduktion in den Folgetagen stimulieren [32, 33] und Produktionsverluste infolge Trockenheit beziehungsweise Dürre, zumindest teilweise, kompensieren. Kompensation könnte die Stabilisierung der GräserSPIn während der jüngeren Klimaerwärmung erklären. Die Blühdauer der Gräser blieb über die Jahre relativ konstant. Dementsprechend hatte der frühe Blühbeginn der Gräser 2018 auch ein früheres Blühende zur Folge. Dies entspricht der Blühphasen-Hypothese (siehe oben), dass ein früher Frühling ein frühes Vegetationsende im Herbst nach sich zieht [11].

Trotz Jahreserwärmung im letzten Vierteljahrhundert und gestiegener $\mathrm{CO}_{2}$-Luftkonzentrationen haben die Pollenkonzentrationen von Hasel, Erle, Birke und Gräsern bei Trendanalyse mehrheitlich nicht zu-, sondern abgenommen. Die Zunahme der

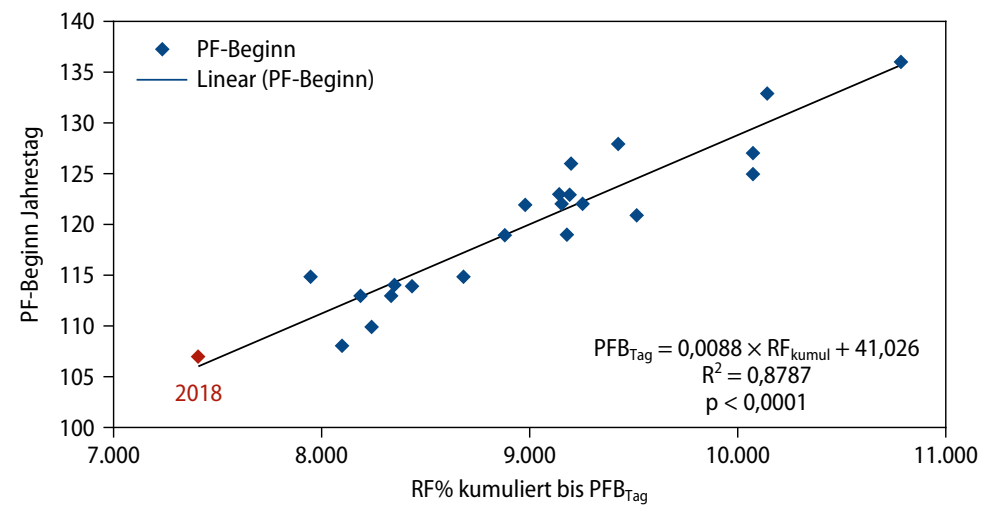

RF, relative Feuchte; PFB/PF-Beginn, Pollenflugbeginn; $M_{T} W_{\text {kumul, }}$ kumulierte mittlere Tageswärme; $N S_{\text {kumu }}$ Tages-NS kumuliert ab 1.1.; $R F_{\text {kumu, }}$ relative Feuchte kumuliert $a b$ 1.1.; Trockenheit (RF-Defizit) verlagert den PFB der Gräser deutlich nach vorne. Bei multivariater Analyse dominiert $R F_{\text {kumul }}$ den $P F B\left(R^{2}=0,8787 ; p=4,37^{*} 10-11\right)$ sosehr, dass die beiden anderen Einflussfaktoren, $M T W_{\text {kumul }}\left(R^{2}=0,317 ; p=0,0051\right)$ und NSkumul $\left(R^{2}=0,338 ; p=0,0036\right)$, unbedeutend werden. Wegen der signifikanten Beziehung zwischen $R F$ und PFB bietet sich $R F_{\text {kumul }}$ als Prädiktor für den PF-Beginn an.

Abb. 8a: Gräser (Poaceae) - relative Feuchte und Pollenflugbeginn 1993 bis 2018 (PF-Beginn-Tag vs. RF\% kumul $_{\text {bis }}$ PF-Beginn)

\section{b}

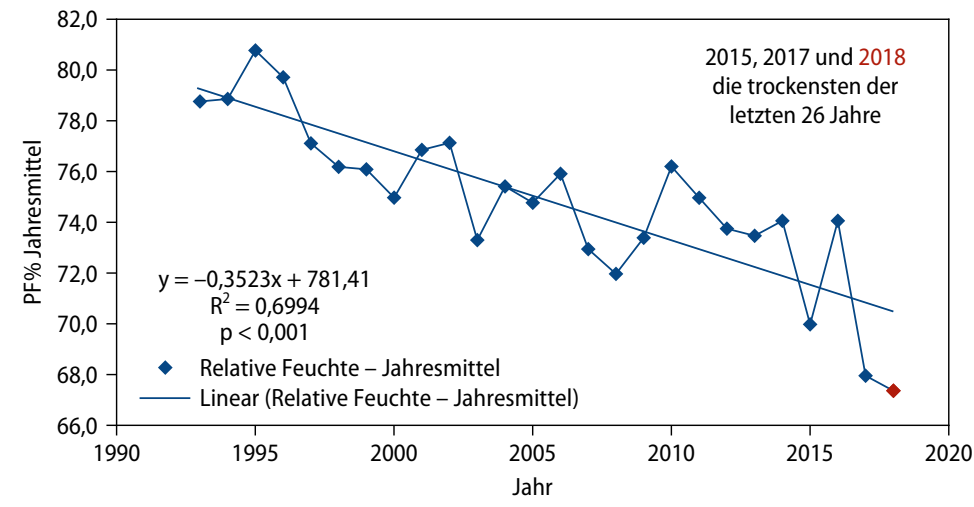

$R F$, relative Feuchte;

Auffällig ist die kontinuierliche Abnahme der relativen Feuchte. Diese Entwicklung geht in Richtung semi-arides Klima.

Abb. 8b: Relative Feuchte im Jahresmittel von 1993 bis 2018 (RF-Abnahme entspricht Feuchtedefizit bzw. Lufttrockenheit.)

Pollenallergie sowie der Prävalenz der Sensibilisierung vor allem bei jungen Menschen [34] können damit nicht unmittelbar auf die Klimaerwärmung zurückgeführt werden, sondern müssen in anderen Ursachen gesucht werden. Dafür kommen mehrere Faktoren beziehungsweise Faktorenkomplexe infrage: aerogene, zum Beispiel Luftverunreinigung, Allergenalteration sowie toxische oder kombiniert toxisch-allergische Effekte auf Menschen und Pflanzen, die wiederum selbst in Zusammenhang mit der Klimaveränderung stehen können, etwa $\mathrm{NOx}, \mathrm{O}_{3}$, VOC (Stickstoffoxide, Ozon, volatile organische 
Komponenten) $[35,36,37]$, oder ein urbaner Lebensraum. Daneben ist auch eine Reihe anderer non-aerogener Sensibilisierungs- und Reaktionsverstärker wahrscheinlich.

\section{Dr. Herwig AE Schinko}

Pneumologie

Allgemeines Krankenhaus der Stadt Linz

Kepler Universitätsklinikum, Med Campus III

Bergweg 16

A-4203 Altenberg/Linz

Österreich

E-Mail: herwig@schinko.co.at

\section{Danksagung}

Wir danken der ZAMG Salzburg-Wien (J. Haslhofer) und der Abteilung Umweltschutz des Amtes der OÖ Landesregierung (R. Pürmayr) für die Bereitstellung meteorologischer Daten; dem Umweltmanagement des Magistrats der Landeshauptstadt Linz (W. Hager) ebenfalls für die Bereitstellung meteorologischer Werte; den Betreibern der EAN-Datenbank des Österreichischen Pollenwarndienstes an der HNO-Klinik der Medizinischen Universität Wien (U. Berger, K. Bastl und M. Bastl); dem technischen Team des Pollenwarndienstes am Kepler Universitätsklinikum Linz (J. Brungraber, P. Lichtenberger, A. Maschik); der Pollenanalytik in Mondsee (J. Schmidt); sowie für ihre Anregungen Ch. Körner (Basel), A. Pauling (Zürich), H. Formayer (Wien).

\section{Interessenkonflikt}

R. Schmidt ist seit 32 Jahren als Pollenanalytiker für den Österreichischen Pollenwarndienst tätig; dadurch entsteht jedoch kein Interessenkonflikt mit der präsentierten Arbeit.

B. Lamprecht und $\mathrm{H}$. AE Schinko geben an, dass keine Interessenkonflikte vorliegen.

\section{Zitierweise}

Schinko HAE, Lamprecht B, Schmidt R. How will climate change alter the dynamics of airborne pollen and pollen load of allergenic plants? The "exceptionally warm year" 2018 in Linz (Upper Austria) - a potential model for the future based on long-term trends. Allergo J Int 2021; 30:96-108

https://doi.org/10.1007/s40629-020-00152-4

\section{Literatur}

1. Zwick H, Popp W, Jäger S, Wagner C, Reiser K, Horak F. Pollen sensitization and allergy in children depend on the pollen load. Allergy 1991;46:362-6

2. Popp W, Zwick H, Steyrer K, Rauscher H, Wanke T. Sensitization to aeroallergens depends on environmental factors. Allergy 1989;44:572-5

3. Stemeseder T, Klinglmayr E, Moser S, Lüftenegger L, Lang $\mathrm{R}, \mathrm{Himly} \mathrm{M}$ et al. Cross-sectional study on allergic sensitization of Austrian adolescents using moleculebased IgE profiling. Allergy 2017;72:754-63

4. Austrian Panel on Climate Change (APCC). Austrian Special Report Health, Demography and Climate Change (ASR18). Haas W, Moshammer H, Muttarak R, Koland O, eds. Wien: Austrian Academy of Sciences Press 2018, p. 331 [URL: https://austriaca.at/APCC_ASR18.pdf]

5. Ziello C, Sparks TH, Estrella N, Belmonte J, Bergmann KC, Bucher $E$ et al. Changes to airborne pollen counts across Europe. PLoS One 2012;7:e34076
6. Bergmann KC. Weather conditions and climate change have an effect on allergies. Allergo J Int 2016;25:131-7

7. Zentralanstalt für Meteorologie und Geodynamik (ZAMG). 2018 mit großer Wahrscheinlichkeit wärmstes Jahr der Messgeschichte. News vom 20.12.2018 (www. zamg.ac.at/cms/de/klima/news/2018-mit-grosser-wahrscheinlichkeit-waermstes-jahr-der-messgeschichte]und [URL: www.zamg.at/histalp)

8. Kromp-Kolb H, Formayer H, Haas P, Hofstätter M, Schwarzl I. Beobachtete Veränderung der Hitzeperioden in Oberösterreich und Abschätzung der möglichen zukünftigen Entwicklungen (Endbericht Band 1 der Forschungsreihe "Auswirkungen des Klimawandels auf Oberösterreich", Februar 2007). BOKU-Met Report 12 2009, pp. 1-31 (https://meteo.boku.ac.at/report/BOKUMet Report 12 online.pdf)

9. Menzel A, Sparks TH, Estrella N, Koch E, Aasa A, Ahas R et al. European phenological response to climate change matches the warming pattern. Glob Change Biol 2006;12:1969--76

10. Peñuelas J, Rutishauser T, Filella I. Ecology. Phenology feedbacks on climate change. Science 2009;324:887-8

11. Keenan TF, Richardson AD. The timing of autumn senescence is affected by the timing of spring phenology: implications for predictive models. Glob Change Biol 2015;21:2634-41

12. Forkel M, Carvalhais N, Rödenbeck C, Keeling R, Heimann $\mathrm{M}$, Thonicke $\mathrm{K}$ et al. Enhanced seasonal $\mathrm{CO} 2$ exchange caused by amplified plant productivity in northern ecosystems. Science 2016;351:696-9

13. Buermann W, Forkel M, O'Sullivan $M$, Sitch $S$, Friedlingstein $\mathrm{P}$, Haverd $\mathrm{V}$ et al. Widespread seasonal compensation effects of spring warming on northern plant productivity. Nature 2018;562:110-4

14. Bastl K, Kmenta M, Berger UE. Defining pollen seasons: background and recommendations. Curr Allergy Asthma Rep 2018;18:73

15. Fritz A. Der Pollen- und Sporenflug in Kärnten 1983 und 1984. Carinthia II 1985;175/95:111-24 (www.zobodat.at/ pdf/CAR_175_95_0111-0124.pdf)

16. Jäger $S$. Tageszeitliche Verteilung und langjährige Trends bei allergiekompetenten Pollen. Allergologie 1990;13:159-82

17. Zwander H, Fischer-Wellenborn E, Romauch E. Der Pollenflug in Kärnten im Jahr 2001. Carinthia II 2002;192/112:141-53 (www.zobodat.at/pdf/ CAR 192 112 0141-0153.pdf)

18. Schmidt R, Schmidt J, Schinko HAE. Aerobiologie Erfahrungen aus 25 Jahren Pollenflug Linz. In: Schinko HAE, Schmidt R, Lamprecht B, eds. Pollen, der Mensch und die Stadt. Aeroallergie \& Aerobiologie, Interdisziplinäres Symposium 2011. Eigenverlag: Linz 2011, pp. 7-28

19. Laube J, Sparks TH, Estrella N, Höfler J, Ankerst DP, Menzel A. Chilling outweighs photoperiod in preventing precocious spring development. Glob Change Biol 2014;20:170-82

20. Pauling A, Gehring R, Clot B. Toward optimized temperature sum parameterizations for forecasting the start of the pollen season. Aerobiologia 2014;30:45-57

21. Körner C, Basler D. Plant science. Phenology under global warming. Science 2010;327:1461-2

22. Barnes C, Pacheco F, Landuyt J, Hu F, Portnoy J. The effect of the temperature, relative humidity and rainfall in airborne ragweed pollen concentrations. Aerobiologia 2001;17:61-8

23. Jäger $S$, Hirnschall I, Hürbe $E$, Schmidt R, Wiesenberger W. Pollenwarndienst des Landes Oberösterreich (LKH Gmundnerberg) und der Stadt Linz (AKh Linz). Mitt Ärztekammer Oberösterreich 1985;81:426-9

24. Galán C, Smith M, Thibaudon M, Frenguelli G, Oteros J, Gehrig R et al. EAS QC Working Group. Pollen monitoring: 
minimum requirements and reproducibility of analysis. Aerobiologia 2014;30:385-95

25. Galán C, Ariatti A, Bonini M, Clot B, Crouzy B et al. Recommended terminology for aerobiological studies. Aerobiologia 2017;33:293-5

26. Kmenta M, Bastl K, Kramer MF, Hewings SJ, Mwange J, Zetter $\mathrm{R}$ et al. The grass pollen season 2014 in Vienna: A pilot study combining phenology, aerobiology and symptom data. Sci Total Environ 2016;566-567:1614-20

27. Schaumberger A. Räumliche Modelle zur Vegetationsund Ertragsdynamik im Wirtschaftsgrünland. Diss. Technische Universität Graz 2011, 264 S.

28. Medhaug I, Stolpe MB, Fischer EM, Knutti R. Reconciling controversies about the 'global warming hiatus'. Nature 2017;545:41-7

29. Lenz A, Hoch G, Vitasse Y, Körner C. European deciduous trees exhibit similar safety margins against damage by spring freeze events along elevation gradients. New Phytol 2013;200:1166-531

30. Masaka K, Maguchi S. Modelling the masting behaviour of Betula platyphylla var. japonica using the resource budget model. Ann Bot 2001;88:1049-55

31. Haslinger K, Schöner W, Anders I. Future drought probabilities in the Greater Alpine Region based on COSMO-CLM experiments - spatial patterns and driving forces. Meteorol Z 2015;25:137-48
32. Spieksma FTM, den Tonkelaar, JF. Four-hourly fluctuations in gras-pollen concentrations in relation to wet versus dry weather, and to short versus long over-land advections. Int J Biometeorol 1986;30:351-8

33. Munoz Rodríguez AF, Palacios I, Molina, R. Influence of meterological parameters in hourly patterns of grass (Poaceae) pollen concentrations. Ann Agric Environ Med 2010;17:87-100

34. Haftenberger M, Laußmann D, Ellert U, Kalcklösch M, Langen U, Schlaud M et al. Prävalenz von Sensibilisierungen gegen Inhalations- und Nahrungsmittelallergene Ergebnisse der Studie zur Gesundheit Erwachsener in Deutschland (DEGS1). Bundesgesundheitsbl 2013;56:68797

35. Schinko HAE, Medinger W, Hager W. Pollen, Pollenallergene und partikuläre Luftschadstoffe Aspektewandel. Allergologie 1994;17:514-25

36. Sénéchal H, Visez N, Charpin D, Shahali Y, Peltre G, Biolley $J P$ et al. A review of the effects of major atmospheric pollutants on pollen grains, pollen content, and allergenicity. ScientificWorldJournal 2015;2015:940243

37. Wooding DJ, Ryu MH, Hüls A, Lee AD, Lin DTS, Rider CF et al. Particle depletion does not remediate acute effects of traffic-related air pollution and allergen. A randomized, double-blind crossover study. Am J Respir Crit Care Med 2019;200:565-74 